\title{
Fuzzy Approach for Sentiment Analysis
}

\author{
Chris Jefferson, Han Liu, IEEE and Mihaela Cocea, IEEE \\ School of Computing, University of Portsmouth, Portsmouth, United Kingdom \\ Emails: Chris.Jefferson@myport.ac.uk, \{Han.Liu, Mihaela.Cocea\}@port.ac.uk
}

\begin{abstract}
Sentiment analysis aims to identify the polarity of a document through natural language processing, text analysis and computational linguistics. Over the last decade, there has been much focus on sentiment analysis as the data available on-line has grown exponentially to include many sentiment based documents (reviews, feedback, articles). Many approaches consider machine learning techniques or statistical analysis, but there has been little use of the fuzzy classifiers in this field especially considering the ambiguity of language and the suitability of fuzzy approaches to deal with this ambiguity. This paper proposes a fuzzy rule based system for sentiment analysis, which can offer more refined outputs through the use of fuzzy membership degrees. We compare the performance of our proposed approach with commonly used sentiment classifiers (e.g. Decision Trees, Naïve Bayes) which are known to perform well in this task. The experimental results indicate that our fuzzybased approach performs marginally better than the other algorithms. In addition, the fuzzy approach allows the definition of different degrees of sentiment without the need to use a larger number of classes.
\end{abstract}

Keywords -Data Mining; Machine Learning; Fuzzy Rule Based Systems; Text Classification; Sentiment Analysis

\section{INTRODUCTION}

With increasing on-line stores of opinion based documents (movie reviews, product feedback, etc.), a large area of research and analysis has grown up around them [1]. This had created a growth area for tasks such as polarity classification, agreement detection and analysis of the degree of positivity related to the polarity of documents such as those mentioned above [2]. This level of analysis is useful as it allows for a high level summary of the tone of a document to be identified, through the use of different machine learning and natural language processing techniques. However, this disregards the fact that a document may be made up of aspects linking it to multiple sentiments or opinions, and this highlights a limitation of how many classifiers are being applied to the sentiment analysis field. To address this issue, we propose a fuzzy rule based system for classification of sentiment.

Sentiment analysis (also known as opinion mining) is the process by which text is analysed to extract opinion and assign a relevant sentiment, usually positive, negative or neutral. The process uses natural language processing through a combination of pre-processing steps, filtering and stemming techniques, combined with relevant statistical and machine learning classification techniques [1],[2].

Common machine learning techniques such as Naïve Bayes [3], support vector machines (SVM) [4] and decision trees [5] are typically used as classifiers for identifying sentiment or opinion, and assigning a label (typically positive or negative) to a piece of text. These classifiers treat the classification of sentiment in a "black-and-white" manner, while in reality sentiment is rarely clear-cut. In contrast to these, our proposed fuzzy approach can extract the degree to which a document contains a specific sentiment. Based on the predicted classes (e.g. positive and negative) and the corresponding fuzzy membership values, the proposed approach allows the inference of more refined categories (e.g. neutral) or intensities (e.g. somewhat positive, somewhat negative) of sentiment without the need to define more classes.

We compare the performance of our proposed fuzzy approach with the common techniques used in sentiment analysis mentioned above. As SVM models have limitations in transparency [6], we will use Naïve Bayes and decision trees.

The remainder of the paper is structured as follows: Section II covers related studies that outline previous work on sentiment analysis. Section III outlines the proposed approach and its suitability for sentiment analysis. Section IV covers the experimental setup through the use of the Knime platform [7] to compare the fuzzy classifier with a decision tree and a naïve Bayes classifier. Section V presents the results and outlines how different degrees of sentiment can be defined based on fuzzy membership degree values. Section VI concludes the paper and outlines further research in this area.

\section{RELATED STUDIES}

In this section we give an overview of previous work related to sentiment analysis, including types of classification tasks, types of data, pre-processing techniques and machine learning algorithms. We also give a comprehensive overview of fuzzy approaches for text processing.

Most approaches in the sentiment analysis area focus on polarity or opinion classification into positive or negative classes [8], [9], [10]; some researchers also include a neutral class [11], [12]. Other classification tasks focus on subjectivity vs. objectivity [13], on predicting categories of emotions (e.g. anger, fear joy) [14], [15] or the strength of sentiment [16].

The research in this area has been applied to a variety of data sources, such as movie reviews [10], product reviews [17], [18], Facebook data [11] and micro-blog data [12], [19]. In this research reported in this paper we use movie reviews data.

To prepare the data for classification tasks, several preprocessing techniques are typically used, such as spelling corrections, tokenisation (splitting the text in tokens such as 
words), and removal of numbers, punctuation and repeated letters, e.g. [6], [11], [12].

Machine learning approaches have been successfully used for sentiment analysis and a number of algorithms have been shown to perform well for sentiment analysis tasks: Naïve Bayes, Support Vector Machines, Maximum Entropy and Decision Trees [20], [21].

In recent years, fuzzy approaches have started to emerge for text processing. In 2012, a review of fuzzy approaches for natural language processing [22] highlighted that the percentage of papers relating to fuzzy approaches is very low over all the papers in the literature of natural language processing despite the suitability of fuzzy approaches for text processing and classification. Since then, a number of fuzzy approaches have been proposed for a variety of applications, as outlined below.

A fuzzy fingerprint text based classification of companies has been developed [23], which outperformed other commonly used non-fuzzy techniques. A fuzzy approach was used in [24] to automatically build a corpus to be used for text similarity comparison; their results showed that the fuzzy metrics had a higher correlation with human ratings when compared with traditional metrics. An unsupervised fuzzy approach [25] was used to classify Twitter users according to gender. A fuzzy rule based approach was proposed in [26], which was shown to lead to a reduction in computational complexity while maintaining a similar performance to other well-known machine learning approaches.

In this paper we build on the work in [26] to discuss how the membership degree values can be used for more refined outputs, including different intensities or strengths of sentiment. We compare the performance of the fuzzy approach with machine learning algorithms known to perform well on sentiment analysis tasks. Unlike previous approaches, we do not only look at the classification performance, but at ways in which fuzzy approaches can be used to provide more refined, interpretable outputs.

\section{PROPOSED APPROACH}

We propose the use of fuzzy rule based systems for sentiment classification. This section presents the key features of the fuzzy approach and justifies its significance in practical applications for sentiment classification.

\section{A. Key Features}

In general, there are three popular types of fuzzy rule based systems, namely, Mamdani, Sugeno and Tsukamoto [27]. The first two types apply to regression problems, since the outputs derived from these two types of fuzzy systems are real values. The third type of fuzzy systems generally applies to classification problems, since the outputs derived by this type of fuzzy systems are discrete values. Therefore, the proposed approach involves the use of the Tsukamoto fuzzy rule based systems for sentiment classification.
Learning of a fuzzy rule based system typically involves building a number of fuzzy membership functions for each continuous attribute, i.e. each continuous attribute is divided into a number of linguistic attributes, each of which is with a specified fuzzy membership function and the values of which are fuzzy membership degrees ranging from 0 to 1 . Membership functions can be of various types, such as trapezoidal, triangular and rectangular [27]. In this paper, we use the trapezoidal membership function, since it is one of the most popular ones [28] and can be seen as a generalization of triangular and rectangular membership functions. In fact, defining a trapezoidal membership function is essentially aimed at specifying four parameters $a, b, c, d$ for a linguistic attribute $T$. An example is illustrated in Eq. (1) and in Fig. 1. In this example, the fuzzy membership function would be triangular if $b=c$. Also, the fuzzy membership function would be rectangular if $a=b$ and $b=c$. Therefore, $a$ trapezoidal membership function is viewed as a generalization of triangular and rectangular membership functions and thus used in this paper.

$$
f_{T}(x)=\left\{\begin{array}{l}
0, x \leq a \\
(x-a) /(b-a), a \leq x \leq b \\
1, b \leq x \leq c \\
(d-x) /(d-c), c \leq x \leq d \\
0, x \geq d
\end{array}\right.
$$

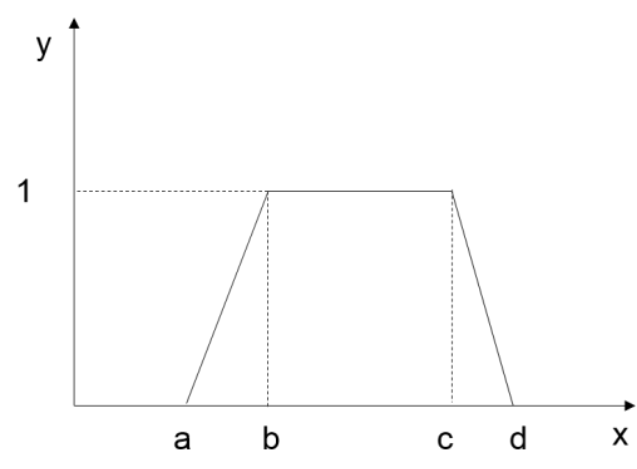

Fig. 1. Trapezoid Fuzzy Membership Function [26]

In the training stage, the values of the four parameters $a, b$, $\mathrm{c}, \mathrm{d}$ are derived for each linguistic attribute transformed from a continuous attribute, such that fuzzy rules are generated. In the testing stage, fuzzy rule based classification involves five main operations: fuzzification, application, implication, aggregation and defuzzification [27]. For example, a rule base consists of four fuzzy rules as follows:

Rule1: If $\mathrm{x}_{1}$ is Good and $\mathrm{x}_{2}$ is High Then class= Positive;

Rule2: If $\mathrm{x}_{1}$ is Good and $\mathrm{x}_{2}$ is Low Then class= Positive;

Rule3: If $\mathrm{x}_{1}$ is $\mathrm{Bad}$ and $\mathrm{x}_{2}$ is High Then class= Negative;

Rule4: If $\mathrm{x}_{1}$ is $\mathrm{Bad}$ and $\mathrm{x}_{2}$ is Low Then class $=$ Negative.

For this example, if we suppose $a=2, b=6, c=9$ and $d=9$ for the fuzzy membership functions defined for both 'Good' 
and 'High', and let $\mathrm{x}_{1}=3$ and $\mathrm{x}_{2}=5$, then the above five operations would be executed in the following procedure:

Fuzzification:

Rule 1: $f_{\text {Good }}(3)=0.25, f_{\text {High }}(5)=0.75$;

Rule $2: f_{\text {Good }}(3)=0.25, f_{\text {Low }}(5)=0.25$;

Rule 3: $f_{\text {Bad }}(3)=0.75, f_{\text {High }}(5)=0.75$;

Rule 4: $f_{\text {Bad }}(3)=0.75, f_{\text {Low }}(5)=0.25$;

In the fuzzification stage, the aim is to map the real value of a numerical attribute to a membership degree to a fuzzy set labelled with a linguistic term. In particular, the notation $f_{\text {Good }}(3)$ indicates that the membership degree of the real value ' 3 ' to the linguistic term 'Good' is 0.25. Similarly, the notation $f_{H i g h}(5)$ indicates that the membership degree of the real value ' 5 ' to the linguistic term 'High' is 0.75 .

\section{Application:}

Rule 1: $f_{\text {Good }}(3) \wedge f_{\text {High }}(5)=\operatorname{Min}(0.25,0.75)=0.25$;

Rule 2: $f_{\text {Good }}(3) \wedge f_{\text {Low }}(5)=\operatorname{Min}(0.25,0.25)=0.25$;

Rule 3: $f_{\text {Bad }}(3) \wedge f_{\text {High }}(5)=\operatorname{Min}(0.75,0.75)=0.75$;

Rule 4: $f_{\text {Bad }}(3) \wedge f_{\text {Low }}(5)=\operatorname{Min}(0.75,0.25)=0.25$;

In the application stage, the aim is to derive the firing strength of each fuzzy rule through the conjunction of the two membership degrees respectively for the two numerical attributes $\mathrm{x}_{1}$ and $\mathrm{x}_{2}$.

Implication:

Rule 1: $f_{l}($ Positive $)=\operatorname{Min}(0.25,1)=0.25$

Rule 2: $f_{2}$ (Positive $)=\operatorname{Min}(0.25,1)=0.25$;

Rule 3: $f_{3}$ (Negative $)=\operatorname{Min}(0.75,1)=0.75$;

Rule 4: $f_{4}($ Negative $)=\operatorname{Min}(0.25,1)=0.25$;

In the implication stage, the firing strength of a fuzzy rule derived in the application stage is used further to determine the membership degree of the value of the class attribute ' $y$ ' to the linguistic term 'Positive' or 'Negative', depending on the consequent of the fuzzy rule. For example, $f_{l}$ (Positive $)=0.25$ indicates that the consequent of Rule 1 is 'Positive' and the value of the class attribute ' $y$ ' belongs to 'Positive' with the membership degree of 0.25 . Similarly, $f_{4}$ (Negative) indicates that the consequent of Rule 4 is 'Negative' and the value of the class attribute ' $y$ ' belongs to 'Negative' with the membership degree of 0.25 .

Aggregation:

$$
\begin{aligned}
f(\text { Positive }) & =f_{1}(\text { Positive }) \vee f_{2}(\text { Positive }) \\
& =\operatorname{Max}(0.25,0.25)=0.25 \\
f(\text { Negative }) & =f_{3}(\text { Negative }) \vee f_{4}(\text { Negative }) \\
& =\operatorname{Max}(0.75,0.25)=0.75
\end{aligned}
$$

In the aggregation stage, the value of the class attribute ' $y$ ' derived from each rule needs to have its membership degree to the corresponding linguistic term taken towards finding the maximum among all the membership degrees. For example, Rule 1 and Rule 2 both provide 'Positive' as the linguistic output and the values of the class attribute ' $y$ ' derived through the two rules both have the membership degree of 0.25 to the linguistic term 'Positive'. Therefore, the value of the class attribute is considered to have the membership degree of 0.25 to 'Positive'. Similarly, the maximum of the membership degrees derived through Rule 3 and Rule 4 is 0.75 , so the value of the class attribute is considered to have the membership degree of 0.75 to 'Negative'.

Defuzzification:

$f($ Negative $)>f($ Positive $)=>$ class $=$ Negative

The defuzzification stage aims to identify the linguistic term to which the value of the class attribute has the highest membership degree. In this example, the value of the class attribute has the membership degree of 0.75 to the linguistic term 'Negative', which is higher than the membership degree of 0.25 to the other linguistic term 'Positive', so the final output is 'Negative' towards classifying an unseen instance.

\section{B. Justification}

We propose the use of fuzzy rule learning approach due to its nature of learning and the advantages of fuzzy logic, as well as its suitability for the problem as outlined below.

Firstly, fuzzy logic is well capable of dealing with linguistic uncertainty. In particular, it considers a classification problem to be a 'degree of grey' problem rather than a 'black and white' problem (currently used in sentiment analysis). This way of defining the problem leads to a reduction of bias on both positive and negative sides. For example, popular algorithms for sentiment classification, such as C4.5 (a type of decision tree) and Naive Bayes, deal with continuous attributes by discretising numerical values (e.g. word frequency in sentiment analysis) into different intervals, each of which is used as a condition judgement towards the final classification. This way of handling continuous attributes has been generally criticised as judgement bias in fuzzy logic literature. In particular, Zadeh argued in [29] that the transition from membership to non-membership is actually gradual rather than sharp. The judgement bias can be resolved by using fuzzy linguistic attributes instead, since each linguistic attribute is a fuzzy set with unsharp boundaries regarding the membership or non-membership of an element to a set. In addition, through the use of fuzzy logic, the classification outcome is provided with a degree to which an instance belongs to each class.

Secondly, fuzzy rule learning approaches are fundamentally different from those learning approaches popularly used in sentiment classification, such as support vector machine, Naïve Bayes and C4.5. These popular approaches all belong to discriminative learning, i.e. the aim of learning is to discriminate one class from all other classes leading to a single class being assigned uniquely to a test instance. This type of learning is based on the assumption that different classes are mutually exclusive so it is very sensitive to the class imbalance problem and cannot deal effectively with fuzziness in text instances.

In contrast, fuzzy approaches typically belong to generative learning, i.e. the aim of learning is to judge the membership degree of an instance to each single class. This 
type of learning is much less sensitive to class imbalance since the learning objective can be simply at inducing the fuzzy relationships between input attributes and classes by learning from each class of instances separately, without the need to discriminate between classes. In this context, it may appear to have the phenomenon that an instance has the membership degree of 1 to multiple classes, i.e. it belongs to more than one class, or that an instance has the membership degree of 0 to all the classes, i.e. it does not belong to anyone of the classes and is thus unclassified. Thus, overall, fuzzy approaches can deal more effectively with fuzziness in text instances.

\section{EXPERIMENTAL SETUP}

This section outlines the experimental setup used to analyse the feasibility of using a fuzzy rule based classifier for obtaining degrees of sentiment. This includes identification of relevant datasets, data pre-processing, model building and evaluation in the Knime platform [7].

In this study we used a total of five datasets from two sources. The first four datasets that were utilised are the Sentiment movie datasets extracted from IMDB by Pang and Lee [30]. These use collections of movie-review documents labelled with respect to their overall sentiment polarity, i.e. positive or negative.

The fifth dataset used is an extension on the dataset used in [10], in which the 2 polarity classes (positive/ negative) are replaced with 5 degrees of sentiments, i.e. negative, somewhat negative, neutral, somewhat positive and positive. The dataset is hosted by Kaggle [31] as part of a competition into sentiment analysis that was based on works of [32].

We refer to the first four datasets as dual sentiment datasets, and the fifth one as multi-sentiment to highlight the difference in the number of classes used.

To prepare the data for the experiments we performed typical pre-processing tasks, as outlined below:

- Using taggers [33]: this step involves processing the document so that parts of speech and relevant phrases are tagged correctly as single entities. This tagging process is broken down into two tasks. The first task is the tagging of parts of speech using the Penn Treebank tag set to mark-up the parts of speech and then an Abner tagger is used to supplement the document tagging.

- Once the tagging is completed, the bag of words method [34] is used to identify which documents contain specific words and entities. This is represented as a tuple of the words contained within the document to provide relevant ranking information for counting term frequency.

- Frequency calculation is performed next with the preprocessing calculating the term frequency (both relative and non-relative), the inverse category frequency and then the inverse document frequency.

- Filters are used to ensure the kept terms meet the frequency requirement (in this case 1000) at the inverse document frequency count. Next, punctuation and numbers are filtered out. Finally stop words are removed using a stop word filter that relies on a built-in dictionary.
- Stemming [33] is applied to ensure that all words are reduced to their root to avoid ambiguous classification.

- A document vector [33] is associated to the relevant data elements representing the feature sets of each document.

These pre-processing steps will lead to a repeatable approach with different data sets, and enable consistent results for each classifier. These stand as the first four of the five stages of sentiment analysis (enrichment, transformation, preprocessing, vectoring and mining) [33].

To assess the performance of the proposed fuzzy approach we compare it with algorithms known to perform well in sentiment analysis tasks, namely Naïve Bayes and Decision Trees. While SVM also performs well in sentiment analysis tasks, we excluded it from our experiments due to its lack of transparency.

For the evaluation of the results, we used two setups: (a) training-testing split, with two thirds of the data for training and one third for testing; (b) 10-fold cross validation.

\section{RESUltS AND DisCUSSION}

In this section we present the results of the three classifiers on the datasets mentioned in Section IV. We discuss the performance, as well as how the fuzzy membership degree values can be used for more refined outputs.

The performance results on the dual sentiment datasets are displayed in Table I. Overall, all classifiers achieve a good performance with an accuracy of over 0.9 ; there are some small differences between the classifiers, with each of them performing slightly better than the other two for different datasets and evaluation setup.

TABLE I. ACCURACY ReSUltS For DUAL DATASETS

\begin{tabular}{|c|c|c|c|}
\hline Dataset & $\begin{array}{l}\text { Evaluation } \\
\text { Setup }\end{array}$ & Classifier & Accuracy \\
\hline \multirow{6}{*}{$\begin{array}{l}\text { Dual Dataset } \\
\text { v2.0 }\end{array}$} & \multirow[t]{3}{*}{ Training Testing } & Decision Tree & 0.929 \\
\hline & & Fuzzy Rules & 0.929 \\
\hline & & Naïve Bayes & 0.913 \\
\hline & \multirow[t]{3}{*}{ Cross Validation } & Decision Tree & 0.923 \\
\hline & & Fuzzy Rules & 0.938 \\
\hline & & Naïve Bayes & 0.926 \\
\hline \multirow{6}{*}{$\begin{array}{l}\text { Dual Dataset } \\
\text { v0.9 }\end{array}$} & \multirow[t]{3}{*}{ Training Testing } & Decision Tree & 0.942 \\
\hline & & Fuzzy Rules & 0.948 \\
\hline & & Naïve Bayes & 0.942 \\
\hline & \multirow[t]{3}{*}{ Cross Validation } & Decision Tree & 0.946 \\
\hline & & Fuzzy Rules & 0.943 \\
\hline & & Naïve Bayes & 0.945 \\
\hline \multirow{6}{*}{$\begin{array}{l}\text { Dual Dataset } \\
\text { v1.1 }\end{array}$} & \multirow[t]{3}{*}{ Training Testing } & Decision Tree & 0.951 \\
\hline & & Fuzzy Rules & 0.956 \\
\hline & & Naïve Bayes & 0.959 \\
\hline & \multirow[t]{3}{*}{ Cross Validation } & Decision Tree & 0.950 \\
\hline & & Fuzzy Rules & 0.948 \\
\hline & & Naïve Bayes & 0.948 \\
\hline \multirow{6}{*}{$\begin{array}{l}\text { Dual Dataset } \\
\text { v1.0 }\end{array}$} & \multirow[t]{3}{*}{ Training Testing } & Decision Tree & 0.930 \\
\hline & & Fuzzy Rules & 0.933 \\
\hline & & Naïve Bayes & 0.930 \\
\hline & \multirow[t]{3}{*}{ Cross Validation } & Decision Tree & 0.945 \\
\hline & & Fuzzy Rules & 0.945 \\
\hline & & Naïve Bayes & 0.950 \\
\hline
\end{tabular}


TABLE II. MEAN ACCURACY STATISTICS ACROSS MULTI SENTIMENT DATASET

\begin{tabular}{|l|l|l|c|}
\hline \multicolumn{1}{|c|}{ Dataset } & $\begin{array}{l}\text { Evaluation } \\
\text { Setup }\end{array}$ & Classifier & Accuracy \\
\hline \multirow{3}{*}{$\begin{array}{l}\text { Multi-sentiment } \\
\text { dataset }\end{array}$} & Training Testing & Decision Tree & 0.528 \\
\cline { 3 - 4 } & & Fuzzy Rules & 0.331 \\
\cline { 3 - 4 } & & Naïve Bayes & $\mathbf{0 . 5 6 3}$ \\
\cline { 2 - 4 } & Cross Validation & Decision Tree & 0.551 \\
\cline { 3 - 4 } & & Fuzzy Rules & 0.355 \\
\cline { 3 - 4 } & & Naïve Bayes & $\mathbf{0 . 5 5 9}$ \\
\hline
\end{tabular}

When considering the results across all Dual Sentiment Datasets, the results show that the Fuzzy Classifier performed better on average in terms of prediction accuracy. The difference, however, is very small. Consequently, we can conclude that the proposed fuzzy approach for classification of polarity performs at the same level as the state-of-the art machine learning approaches.

The results for the multi-sentiment dataset are displayed in Table II. Unsurprisingly, the results are much lower across all classifiers. Naïve Bayes performs best, followed by Decision Trees, while the fuzzy approach has the lowest performance.

For the multi-sentiment dataset, new class labels were added to identify different intensities or strengths of sentiment. While this approach may be suitable for discriminative approaches, it leads to overlapping ranges for the fuzzy approach, leading to poor performance.

The proposed fuzzy approach can identify different intensities of sentiment based on the values of the membership degrees without the need to create intermediate classes, as in the multi-sentiment dataset.

Due to the imprecise nature of the fuzzy approach, we can describe a piece of text in terms of both positive and negative sentiment. Thus, we can have a dual output (see column 4 in Table III) that can describe "how much" of the text is positive and "how much" is negative. For the purpose of this analysis we define different intensities as below:

- Values of 0.00: Neutral

- Values between 0.01 and 0.70: somewhat positive/ somewhat negative

- Values between 0.70 and 1.00: positive/negative

Different definition of intensities (i.e. different value intervals) can be used and further research in this area is needed. The definition above is used only to show how the fuzzy degree membership values can be used to define different intensities of sentiment and to analyse what different combinations of values mean for the overall output.

Table III displays different combinations of values for the membership degrees to the negative and positive class (column 1 and 2), a single output, i.e. positive or negative, a dual output, i.e. values for both positive and negative, and sentiment intensities output. For sentiment intensities, we use the categories from the multi-sentiment dataset, i.e. negative, somewhat negative, neutral, somewhat positive and positive.

The first four rows in Table III display situations where one of the two sentiments is dominant (i.e. values over 90\%), with low values for the other sentiment (i.e. values less than $10 \%)$. In this case, the text can be labelled with the dominant class.

Row five in Table III displays a situation when only one sentiment is present in the text - in this case positive; however, the value of the membership degree is around 0.33, indicating a weak membership to the positive class. According to the ranges defined above, this would correspond to "somewhat positive".

Rows six and seven in Table III display situations when both sentiments are present in the text, to different degrees. In row six, the membership to the positive class is high (above 0.70 ), indicating that some of the text is positive; the membership to the negative class is much lower (0.23), but enough to indicate that the text reflects some negative sentiment. As we use the fuzzy approach we can have an output about both classes - in this case it would be "Positive and somewhat negative".

Row seven displays a situation where the text has similar membership degrees to both classes, i.e. around 0.5 , indicating that the text belongs to both classes. In terms of an output reflecting intensities of sentiment, according to the ranges defined previously, this instance is "somewhat positive and somewhat negative". This is in contrast to the discriminative approach, where the aim is to choose between the 2 classes; in this situation a value of $50 \%$ for both the positive and negative class means maximum uncertainty and that the model cannot assign a label, i.e. it will be unclassified.

Row eight in Table III shows what happens when the text does not reflect any sentiment, i.e. membership values of 0.00 for both classes. In this case, for single and dual output, the instance would be unclassified, as it does not belong to any of the classes. For the sentiment intensities output, this instance would be classified as neutral.

TABLE III. DEFINING SENTIMENT INTENSITIES BASED ON FUZZY MEMBERSHIP DEGREES

\begin{tabular}{|c|c|c|l|l|l|}
\hline No & $\begin{array}{c}\text { Degree value for } \\
\text { Class= Negative }\end{array}$ & $\begin{array}{c}\text { Degree Value for } \\
\text { Class= Positive }\end{array}$ & Single output & Dual output & Intensities output \\
\hline 1 & 0.985 & 0.015 & Negative & $98 \%$ Negative and 2\% Positive & Negative \\
\hline 2 & 0.000 & 0.951 & Positive & $0 \%$ Negative and 95\% Positive & Positive \\
\hline 3 & 0.000 & 1.000 & Positive & $0 \%$ Negative and 100\% Positive & Positive \\
\hline 4 & 0.942 & 0.058 & Negative & $94 \%$ Negative and 6\% Positive & Negative \\
\hline 5 & 0.000 & 0.328 & Positive & $0 \%$ Negative and 33\% Positive & Somewhat positive \\
\hline 6 & 0.229 & 0.771 & Positive & $23 \%$ Negative and 77\% Positive & Positive and somewhat negative \\
\hline 7 & 0.529 & 0.471 & Negative & $53 \%$ Negative and 47\% Positive & Somewhat positive and somewhat negative \\
\hline 8 & 0.000 & 0.000 & Unclassified & Unclassified & Neutral \\
\hline
\end{tabular}


From previous approaches on polarity classification, to the best of our knowledge only [16] mentioned a dual output, i.e. a value for each class. They also discuss that a piece of text can contain both positive and negative sentiment at the same time. Thus, more refined outputs, such as the dual output and the intensities output displayed in Table III, are more informative than the typical positive or negative output from machine learning algorithms.

The value of more refined outputs is also reflected in the research direction towards different strengths/intensities of sentiment. To achieve this with current machine learning algorithms based on discriminative learning, more classes are defined. These classes, however, do not have clear boundaries and are not necessarily mutually exclusive, thus breaking the assumptions of many learning algorithms. The fuzzy approach addresses these limitations, allowing more refined outputs without the need to artificially create new classes.

\section{CONCLUSIONS}

In this paper, a fuzzy approach was proposed for sentiment analysis, with a focus on polarity classification. We compared the accuracy of the proposed approach with the accuracy of two other machine learning algorithms (Naïve Bayes and Decision Trees) which are known to be among the best performing techniques for sentiment analysis. The results showed that the proposed approach achieved the same level of performance as the other two algorithms.

In addition to performing well in terms of accuracy, the proposed approach has the advantage of more refined outputs based on the fuzzy membership degree values. We discussed two such outputs: (a) a dual output, with values for both the positive and the negative class and (b) an output reflecting different intensities of sentiment.

In future work, the fuzzy approach for polarity classification will be used for identifying the relationships between classes, through looking at how the membership degrees of different classes are correlated. We will also extend our fuzzy approach for the classification of categories of emotions, an emerging subarea of sentiment analysis.

\section{REFERENCES}

[1] B. Liu, "Sentiment Analysis and Opinion Mining," Morgan \& Claypool, 2012.

[2] E. Cambria, B. Schuller, Y. Xia, and C. Havasi, "New avenues in opinion mining and sentiment analysis," IEEE Intel. Syst, vol. 28, no. 2, pp. 15-21, 2013.

[3] I. Rish, "An empirical study of the naive bayes classifier," IJCAI workshop on empirical methods in artificial intelligence, vol. 3, no. 22, pp. 41-46, 2001.

[4] N. Cristianini and , J. Shawe-Taylor, "An Introduction to Support Vector Machines and Other Kernel-Based Learning Methods," Cambridge: Cambridge University Press, 2000.

[5] J. R. Quinlan "Induction of Decision Trees" Machine learning, vol 1, no 1, pp.81-106, 1986

[6] H. Liu, M. Cocea, and A. Gegov, "Interpretability of computational models for sentiment analysis," in Sentiment Analysis and Ontology Engineering: An Environment of Computational Intelligence, W. Pedrycz and S.-M. Chen, Eds., vol. 639, 2016, pp. 199-220, 2016.

[7] Knime, [Online]. Available: https://www.knime.org/. Accessed: Feb. 7, 2017.

[8] M. Wollmer, F. Weninger, T. Knaup, B. Schuller, S. Congkai, K. Sagae, and L. Morency, "Youtube movie reviews: Sentiment analysis in an audio-visual context," IEEE Intell. Syst., vol. 28, no. 3, pp. 46-53, 2013.
[9] M.-T. Martin-Valdivia, E. Martinez-Camara, J.-M. Perea-Ortega, and L. A. Urena Lopez, "Sentiment polarity detection in Spanish reviews combining supervised and unsupervised approaches," Expert Syst. Appl., vol. 40, pp. 3934-3942, 2013.

[10] B. Pang, L. Lee., and S. Vaithyanathan. "Thumbs Up? Sentiment Classification using Machine Learning Techniques," Proc. ACL-02 conf. on Empirical methods in natural language processing, pp. 79-86, 2002.

[11] A. Ortigosa, J. M. Martin, and R. M. Carro, "Sentiment analysis in Facebook and its application to e-learning," Computers in Human Behavior, vol. 31, pp. 527 - 541, 2014

[12] N. Altrabsheh, M. Cocea, and S. Fallahkhair, "Sentiment Analysis: Towards a Tool for Analysing Real-Time Students Feedback," IEEE 26th Int. Conf. on Tools with Artificial Intelligence, pp. 419-423, 2014.

[13] L. Barbosa and J. Feng, "Robust sentiment detection on twitter from biased and noisy data," Int. Conf. on Computational Linguistics, pp. 36-44, 2010.

[14] I. Perikos and I. Hatzilygeroudis, "Recognizing Emotion Presence in Natural Language Sentences", International Conference on Engineering Applications of Neural Networks, 2013, pp. 30-39.

[15] C Strapparava and R. Mihalcea. "SemiEval-2007 Task14: Affective Text," Proc. 4th Int. Workshop Semantic Evaluations, pp. 70-74, 2007.

[16] M. Thelwall, K. Buckley, and G. Paltoglou, "Sentiment strength detection for the social web," J. Amer. Soc. Inf. Sci. Technol., vol. 63, no. 1, pp. 163-173, 2012.

[17] M. Hu and B. Liu "Minning and Summaizing Customer Reviews", ACM SIGKDD, pp. 168-177, 2004

[18] M. Mostafa, "More than words: Social networks' text mining for consumer brand sentiments," Expert Syst. Appl., vol. 40, pp. 4241-4251, 2013.

[19] R. Prabowo and M. Thelwall, "Sentiment analysis: A combined approach," Journal of Informetrics, vol. 3, no. 2, pp. 143-157, 2009.

[20] B. Pang and L. Lee, "Opinion mining and sentiment analysis," Found. Trends Inf. Retr., vol. 2, nos. 1/2, pp. 1-135, 2008.

[21] N. Altrabsheh, M. Cocea, and S. Fallahkhair, "Learning Sentiment from Students' Feedback for Real-Time Interventions in Classrooms," The Int Conf. on Adaptive and Intelligent Systems - ICAIS, pp. 40-49, 2014

[22] J. Caravalho, F. Batista, and L. Coheur. "A Critical Survey on the use of Fuzzy Sets in Speech and Natural Language Processing", 2012 IEEE Int. Conf. on Fuzzy Systems, pp. 1-8, 2012.

[23] F. Batista and J. P. Carvalho, "Text Based Classification of Companies in CrunchBase," 2015 IEEE Int. Conf. on Fuzzy Systems, pp. 1-7, 2015.

[24] D. Chandran, K. A. Crockett, D. Mclean and A. Crispin, "An automatic corpus based method for a building Multiple Fuzzy Word Dataset," 2015 IEEE Int. Conf. on Fuzzy Systems, 2015, pp. 1-8, 2015.

[25] M. Vicente, F. Batista and J. P. Carvalho, "Twitter gender classification using user unstructured information," 2015 IEEE Int. Conf. on Fuzzy Systems, 2015, pp. 1-7, 2015.

[26] H. Liu and M. Cocea, "Fuzzy Rule Based Systems for Interpretable Sentiment Analysis," The International Conference on Advanced Computational Intelligence, pp. 129-136, 2017.

[27] S. M. Chen, "A fuzzy reasoning approach for rule based systems based on fuzzy logics", IEEE Transactions on Systems, Man and Cybernetics Part B: Cybernetics, vol. 26, no. 5, pp. $769-778,1996$.

[28] T. Ross, "Fuzzy Logic with Engineering Applications". Wiley, 2010.

[29] L. A. Zadeh, "A fuzzy theoretic interpretation of linguistic hedges", Journal of Cybernetics, vol. 2, no. 3, pp. 4-34, 1972.

[30] B. Pang and L. Lee, [Online]. Available: http://www.cs.cornell.edu/ people /pabo/ movie-review-data/. Accessed: Feb. 7, 2017.

[31] Kaggle, [Online]. Available: https://www.kaggle.com/c/sentimentanalysis-on-movie-reviews. Accessed: Feb. 7, 2017.

[32] R. Socher, A. Perelygin, J.Y. Wu, J. Chuang, C.D. Manning, A.Y. Ng, and C. Potts, "Recursive deep models for semantic compositionality over a sentiment treebank," Proc.of EMNLP, pp. 1631-1642, 2013.

[33] K, Tiel and M, Berthold. "The Knime text processing feature: An introduction" KNIME, Tech. Rep, 2012.

[34] K. Reynolds, A. Kontostathis and L. Edwards, "Using Machine Learning to Detect Cyberbullying," Int. Conf. on Machine Learning and Applications and Workshops, pp. 241-244, 2011. 